\title{
Video Article \\ Light-Induced Molecular Adsorption of Proteins Using the PRIMO System for Micro-Patterning to Study Cell Responses to Extracellular Matrix Proteins
}

\author{
Cristina Melero* ${ }^{1}$, Aljona Kolmogorova* ${ }^{1}$, Paul Atherton ${ }^{1}$, Brian Derby ${ }^{2}$, Adam Reid ${ }^{3,4}$, Karin Jansen ${ }^{5}$, Christoph Ballestrem ${ }^{1}$

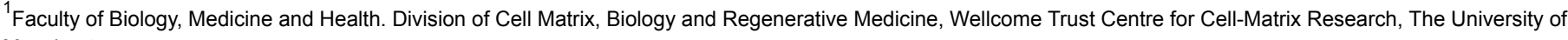 \\ Manchester \\ ${ }^{2}$ School of Materials, The University of Manchester \\ ${ }^{3}$ Blond Mclndoe Laboratories, School of Biological Sciences, Faculty of Biology, Medicine and Health, Division of Cell Matrix Biology and Regenerative Medicine, The \\ University of Manchester, Manchester Academic Health Science Centre \\ ${ }^{4}$ Department of Plastic Surgery \& Burns, Wythenshawe Hospital, Manchester University NHS Foundation Trust, Manchester Academic Health Science Centre \\ ${ }^{5}$ Department of Pathology, UMC Utrecht \\ *These authors contributed equally
}

Correspondence to: Karin Jansen at kjansen6@umcutrecht.nl, Christoph Ballestrem at christoph.ballestrem@manchester.ac.uk

URL: https://www.jove.com/video/60092

DOI: doi:10.3791/60092

Keywords: Immunology and Infection, Issue 152, micro-pattern, patterning, micro-patterning, LIMAP, extracellular matrix (ECM), neuron, cell behavior, migration, axonal growth, pathfinding, decision-making

Date Published: 10/11/2019

Citation: Melero, C., Kolmogorova, A., Atherton, P., Derby, B., Reid, A., Jansen, K., Ballestrem, C. Light-Induced Molecular Adsorption of Proteins Using the PRIMO System for Micro-Patterning to Study Cell Responses to Extracellular Matrix Proteins. J. Vis. Exp. (152), e60092, doi:10.3791/60092 (2019).

\section{Abstract}

Cells sense a variety of extracellular cues, including the composition and geometry of the extracellular matrix, which is synthesized and remodeled by the cells themselves. Here, we present the method of Light-Induced Molecular Adsorption of Proteins (LIMAP) using the PRIMO system as a patterning technique to produce micro-patterned extracellular matrix (ECM) substrates using a single or combination of proteins. The method enables printing of ECM patterns in micron resolution with excellent reproducibility. We provide a step-by-step protocol and demonstrate how this can be applied to study the processes of neuronal pathfinding. LIMAP has significant advantages over existing microprinting methods in terms of the ease of patterning more than one component and the ability to generate a pattern with any geometry or gradient. The protocol can easily be adapted to study the contribution of almost any chemical component towards cell fate and cell behavior. Finally, we discuss common issues that can arise and how these can be avoided.

\section{Video Link}

The video component of this article can be found at https://www.jove.com/video/60092/

\section{Introduction}

In recent years, the biological sciences have increasingly made use of the advances provided by material sciences. One prominent example is the micro-patterning of substrates, which can be used to study cellular responses such as cell proliferation ${ }^{1,2}$, differentiation ${ }^{3,4,5,6}$, cell migration $^{7,8,9}$ and pathfinding ${ }^{10,11}$. There are a number of techniques available that enable the micro-patterning of substrates, such as multiphoton excited photochemistry ${ }^{12}$, AFM dip-pen nanolithography ${ }^{13}$, pin and inkjet direct printing ${ }^{14}$, electron beam lithography ${ }^{15}$ or microfluidics ${ }^{16}$. However, two techniques that are widely used in the biological field are microcontact printing ${ }^{17,18,19}$ or laser-assisted patterning ${ }^{3}$ (Figure 1). Laser-assisted patterning is considered to deliver more reliable results in terms of protein and PEG stability and cell confinement on the patterns, compared to microcontact printing ${ }^{20}$. A more novel approach for micro-patterning described here is the use of Light-Induced Molecular Adsorption of Proteins ${ }^{21}$ (LIMAP, Figure 1D) using a commercially available system (PRIMO, Table of Materials). Each of the methods has advantages and limitations that are briefly described below.Microcontact printing uses PDMS molds (stamps) with desired microfeatures that are generated from lithographed masters. The stamps are incubated with a chosen protein which is then transferred (stamped) onto the cell culture substrate ${ }^{18}$ (Figure 1A). Laser-assisted patterning uses UV light to cleave an anti-fouling film ${ }^{22,23,24,25}$, exposing regions that can subsequently be coated with the protein of interest (Figure 1B). While the resolution achieved with photo-patterning approaches is in the micron range $\mathrm{e}^{25,26}$, most of these techniques require a photo-mask, either in contact with the sample, or situated in the object plane of the microscope objective $e^{23,27,28}$. The requirements for masks in both microcontact printing and photo-patterning can be a limitation; specific masks are required for every geometric pattern and size, which can be expensive and time consuming to generate. In contrast to these techniques, LIMAP does not require a mask (Figure 1D). Using the PRIMO system for LIMAP can be cost intensive at the beginning because it requires purchasing of equipment. However, open-source software is used to design patterns of any desired geometry, giving much more freedom and allowing more complex experiments including the use of protein concentration gradients. The PRIMO laser is controlled and directed by a digitally-controlled micromirror device (DMD) to create patterns in any number of user-defined geometries. LIMAP requires the culture surface to be coated with molecules that prevent cell attachment. Polyethylene glycol (PEG) is most commonly used as such an "antifouling" reagent; it forms a dense 
anti-adhesive film on the glass or plastic surface ${ }^{29}$. Subsequently, a photo-initiator is added that allows the PEG film to be removed with high precision through a photoscission mechanism ${ }^{30}$ by local exposure to UV light under the control of the DMD. These PEG-free regions can be coated with proteins that adsorb to the laser-etched surface, generating a micro-pattern. By varying the laser power, different quantities of PEG can be removed from the surface allowing the user to generate protein gradients. PEG removal and the coating procedure can be repeated to create patterns with two or more distinct proteins in the same micro-well ${ }^{21}$. The generated micro-patterns provide adhesive surfaces for cells, allowing the study of cell behavior. In our studies, we use micro-patterning to study neurite or axon pathfinding of a neuronal cell line (CAD (Cathecholaminergic-a differentiated) cells ${ }^{31}$ ) or primary rat dorsal-root ganglion (DRG) neurons, respectively. Here, we outline a step-by-step protocol for LIMAP (Figure 2) using the commercially available PRIMO system and accompanying Leonardo software. We demonstrate how it can be used for the generation of patterns with defined geometries and multiple proteins, which we use to study axonal pathfinding. We discuss common issues that can arise and how these can be avoided.

\section{Protocol}

\section{Design of pattern templates}

NOTE: Templates for patterning are generated with digital drawing software (Table of Materials). Drawing in different grey levels will determine laser intensities. Using software to design pattern templates allows rapid generation of patterns with any desired geometry and gradients (Figure 3).

1. Digitally draw the desired pattern template using drawing software. Select an image size of 1824 pixels length and 1140 pixels width (which in this study corresponds to $415 \mu \mathrm{m}$ length and $260 \mu \mathrm{m}$ width). Save the pattern template as an 8-bit Tiff file.

NOTE: a step-by-step protocol to generate templates is available upon request to the brand who commercializes the micro-patterning equipment (Table of Materials).

\section{Plasma cleaning}

NOTE: Optimal results require plasma cleaning of the surfaces prior to patterning, which will remove all organic matter and activate the surface. In the present case, ambient air is sufficient for surface activation. A plasma cleaner (Table of Materials) was used with a process pressure of 1000-1300 mTorr and a power of 29.6 W for 1-5 min.

1. Use glass bottom dish/es with a $20 \mathrm{~mm}$ inner well size and glass thickness of $0.16-0.19 \mathrm{~mm}$. For testing multiple conditions, use a 6-well glass bottom dish. Otherwise, use a single well glass bottom dish (Table of Materials).

NOTE: A step-by-step protocol for plasma cleaning is available upon request to the brand who commercializes the plasma cleaning equipment (Table of Materials).

\section{Passivation}

NOTE: This step generates an antifouling film that prevents protein adsorption to the glass surface. PEG offers high resistance to protein adsorption $^{29}$ as an antifouling agent. LIMAP uses a photo-initiator to locally remove PEG through UV photoscission. The protein/s of interest will then adsorb to these PEG-free surfaces ${ }^{21}$, generating micro-patterns.

\section{Passivation with PLL-PEG}

1. Under sterile conditions, cut the PDMS stencils (see Figure 4A,B and Table of Materials) to ensure they fit in the inner glass bottom well, and remove the inner micro-well fillings with sterile forceps. Stick stencils on the glass well using forceps.

2. Ensure that the stencils stick tightly on the glass well, preventing the formation of air bubbles that can cause leakages during the passivation process.

NOTE: PDMS stencils can also be fabricated in-house using published protocols ${ }^{18,32}$

3. Prepare PLL-PEG solution (Table of Materials, $0.1 \mathrm{mg} / \mathrm{mL}$ ) in phosphate-buffered saline (PBS). Add $20 \mu \mathrm{L}$ of PLL-PEG solution to each micro-well and incubate at room temperature, for $1 \mathrm{~h}$.

4. Remove $15 \mu \mathrm{L}$ of PLL-PEG from the micro-wells and wash them five times with $20 \mu \mathrm{L}$ of PBS (Table of Materials) without letting them dry.

NOTE: Always leave approximately $5 \mu \mathrm{L}$ of PBS in between washes. Given their small volume, the micro-wells are particularly susceptible to drying. Drying will result in micro-patterns of poor quality.

5. Either keep the culture dish in PBS ( $3 \mathrm{~mL}$ per well) at $4{ }^{\circ} \mathrm{C}$ for up to 3 days or continue with the next step (step 3.1.5).

6. Remove $18 \mu \mathrm{L}$ of PBS from a single micro-well (for example, the top left micro-well, see Figure 4D), add $5 \mu \mathrm{L}$ of photo-initiator (PLPP, Table of Materials) and leave $20 \mu \mathrm{L}$ of PBS in the remaining micro-wells. This micro-well with PLPP will be used to create a reference pattern (see Figure 4D,E) during the system calibration step (step 4). Keep PLPP protected from light.

\section{Passivation with long-lasting PEG-SVA}

NOTE: To generate the anti-adhesive surface one can use: 1) a PEG linked to Poly-L-Lysine (PLL-PEG, step 3.1) or 2) a PEG-succinate Nhydroxisuccinimide (PEG-SVA). The decision to choose one or the other passivation option depends on the storage options (see step 10). Culture dishes incubated with PEG-SVA require double amount of passivation and photopatterning time.

1. Prepare stencils as described in step 3.1.1.

2. Add $20 \mu \mathrm{L}$ of $0.01 \%$ Poly-L-Lysine (PLL, Table of Materials) to each micro-well and incubate at room temperature for 30 min to precoat with PLL.

3. Remove $15 \mu \mathrm{L}$ of PLL from the micro-wells and wash them three times with $20 \mu \mathrm{L}$ of $1 \mathrm{M}$ HEPES buffer (Table of Materials) without letting the wells dry out. 
NOTE: Always leave approximately $5 \mu \mathrm{L}$ of HEPES or PBS in between washes. Given their small volume, the micro-wells are particularly susceptible to drying. Drying will result in micro-patterns of poor quality.

4. Prepare PEG-SVA solution. PEG-SVA solution $(50 \mathrm{mg} / \mathrm{mL}$ in HEPES buffer $1 \mathrm{M})$ should be prepared fresh each time immediately before use. Prepare $20 \mu \mathrm{L}$ of PEG-SVA per micro-well.

5. HEPES buffer must have a pH between 8-8.5. Test HEPES pH prior to preparing the PEG-SVA solution with pH paper. Weigh PEGSVA in a centrifuge tube using a precision scale. Add HEPES buffer and vortex $30 \mathrm{~s}$ until dissolved. PEG-SVA is completely dissolved when the solution is transparent.

NOTE: SVA is the ester that allows binding of PEG to the previously coated PLL. Once the HEPES buffer is added to the PEG-SVA, the SVA has a half-life of 15 min and should be used immediately.

6. Add $20 \mu \mathrm{L}$ of PEG-SVA solution to each micro-well and incubate at room temperature, for $1 \mathrm{~h}$. Remove $15 \mu \mathrm{L}$ of PEG-SVA from the micro-wells and wash five times with $20 \mu \mathrm{L}$ of PBS (Table of Materials) without letting the wells dry out.

7. Either prepare the culture dish for long-term storage (up to 1 month, see step 10.2) or proceed to the next step (step 3.2.8).

8. Remove $18 \mu \mathrm{L}$ of PBS from a single micro-well (for example, the top left micro-well, see Figure 4D), add $5 \mu \mathrm{L}$ of PLPP (Table of Materials) and leave $20 \mu \mathrm{L}$ of PBS in the remaining micro-wells. This micro-well will be used to create a reference pattern (see Fi gure $4 D, E)$. Ensure that PLPP is homogenous on the whole surface of the micro-well. Keep PLPP protected from light.

\section{System calibration}

NOTE: In these steps, the focus of the laser will be adjusted to the particular type of culture dish (step 4.1). A reference pattern will be generated in only one micro-well (step 4.2) followed by incubation with a protein solution (step 4.3) to ensure the optimal focus conditions of the laser (step 4.4), necessary to obtain sharp and defined patterns.

\section{Laser calibration}

NOTE: During the calibration process, a calibration laser image will be projected onto a fluorescently-highlighted glass surface (calibration well, marked with a fluorescent highlighter, Figure 4C), which needs to be placed in focus on the microscope.

1. Use a fluorescent highlighter (Table of Materials) to mark an empty inner glass well. NOTE: The calibration well must have the same glass thickness $(0.16-0.19 \mathrm{~mm})$ as the culture dish on which micro-patterns will be generated. If a 6-well glass bottom dish is being used, an empty well can be used for calibration and should be marked with fluorescent highlighter under sterile conditions.

2. Switch on the microscope, the stage and the computer. Switch on the PRIMO micro-patterning equipment, open Micro-manager and Leonardo software. Leonardo software is operated through Micro-manager under Plugins. Check the brands/catalogue numbers of equipment and software in Table of Materials.

3. In the initial menu of Leonardo, select Calibrate. Check that the dedicated PRIMO filter cube is in the correct position (optical path) in the filter turret; select the 20X objective (0.75 DIC S Plan Fluor, no phase ring) both on the microscope and on Leonardo software. NOTE: This protocol is adjusted to Leonardo version 4.4. The protocol may need adjusting for other versions.

4. Position the previously highlighted calibration well (Figure 4C) above the objective. Select camera path. Adjust the objective focus until the laser projection of both the PRIMO logo and the tag line Take care of your cells are in focus.

5. Leave default camera exposure time at $25 \mathrm{~ms}$. Adjust the laser intensity to see the letter I from the PRIMO logo projection in grey color and the rest of the letters in white.

6. Record the Z-position of the focal plane, (height of the objective to the sample) later referred to as Z-position of calibration. This will be an approximation to the optimal focus obtained after reference pattern generation (see step 4.2-4.4).

\section{Reference pattern}

1. Position the culture dish with the micro-well containing the photo-initiator (reference pattern micro-well, Figure 4D) above the objective and select Pattern now in the Leonardo software.

2. Visualize the edge of the micro-well with transmitted light through the camera and choose the ROI symbol from the right-hand menu. Set the diameter of the ROI circle to $4000 \mu \mathrm{m}$ and align the edge of the digital ROI with the edge of the current micro-well.

3. Ensure that there is an accurate overlap between the digital ROI and the current micro-well by moving the stage around the edges of the micro-well. The ROI position will be coupled to the stage movements.

4. Select Lock in Leonardo software to lock the ROI in the desired position. Switch off the transmitted light.

5. Select PRIMO to upload the desired pattern template, which will be projected on the ROI as a design unit (see Figure 5). Patterns will appear in a dropdown list referred to as Actions and shown in the Actions menu on the software.

NOTE: Pattern templates need to be designed previously (see step 1) and saved as an 8-bit Tiff file before loading the template in the software.

6. Only a small pattern is needed for the reference pattern; for example, 3 lines, 1 column (see Figure 4E and Figure 5B). In the Replication menu, set the desired number of columns and rows (Lines in Leonardo software). Click Refresh to observe a digital preview of the pattern design.

7. Set the laser dose in the Replication menu. The optimal laser dose in this setting and using PLL-PEG is $1390 \mathrm{~mJ} / \mathrm{mm}^{2}$. NOTE: Laser power can vary between $5-7.5 \mathrm{~mW} / \mathrm{mm}^{2}$. In this case, it is $7.5 \mathrm{~mW} / \mathrm{mm}^{2}$, which takes approximately $30 \mathrm{~s}$ to pattern each design unit, using a laser dose of $1390 \mathrm{~mJ} / \mathrm{mm}^{2}$. Higher laser doses may be required if the culture dish surface is passivated with PEGSVA (approximately double laser dose compared to PLL-PEG). This needs to be tested beforehand.

8. Navigate to a peripheral region of the micro-well, (for example, top part) away from the main region of interest for pattern generation (central region) of the micro-well (see Figure 4E) and select Lock. Wait until the pattern is displayed.

9. Adjust the focus to the Z-position of calibration (see step 4.1.6).

NOTE: It is recommended to do an additional system calibration step if another user is using the same microscope between photopatterning rounds. 
10. Select the Play symbol to start patterning. Ensure the laser is on in the software. Wait until the patterning process is finished. Patterning duration will be displayed in the Estimated Time panel. On Leonardo software version 4.4 patterning is completed when all Actions appear blue in the Visualization menu.

\section{Protein incubation on reference pattern}

1. Under sterile conditions, wash the reference pattern micro-well three times with $20 \mu \mathrm{L}$ of PBS to remove the PLPP.

2. Add $20 \mu \mathrm{L}$ of fluorescently-labeled ECM protein $(10 \mu \mathrm{g} / \mathrm{mL}$ laminin, fibronectin or fibrinogen in PBS, see Table of Materials and step 6) to the reference pattern micro-well. Incubate at room temperature for 10-20 min (depending on the protein) protected from light (wrap the dish in aluminum foil).

3. After incubation, remove $18 \mu \mathrm{L}$ of protein solution and wash three times with $20 \mu \mathrm{L}$ of PBS; keep the micro-wells that are not used to create a reference pattern (see Figure 4D,E) in $20 \mu \mathrm{L}$ of PBS.

\section{Visualization and setting optimal laser focus}

1. Visualize the reference pattern using an epifluorescence microscope, 20X objective and suitable software (check software used in this study in Table of Materials). The reference pattern should be visible in the peripheral region (for example, top well region), in which the reference pattern was generated (see Figure 4E).

2. Adjust focus on the pattern edges through the camera path. Record the Z-position according to the best focus on the reference pattern. This adjusted Z-position will be the optimal laser focus used for subsequent patterning.

\section{Software set-up and photopatterning}

NOTE: Once the calibration of the system has been achieved (step 4), the user will upload the desired pattern templates (template configuration, Figure 5) for photopatterning, with the option to generate patterns for one or multiple proteins in each micro-well. The micro-patterning process involves photopatterning and protein incubation steps (see Figure 2).

1. Under sterile conditions, remove $18 \mu \mathrm{L}$ of PBS from all the micro-wells and add $5 \mu \mathrm{L}$ of PLPP to each micro-well. Ensure that PLPP is homogenous on the whole surface of the micro-wells.

2. Position the culture dish with the reference pattern micro-well (Figure 4D,E) above the objective and select Pattern now in the Leonardo software.

3. Visualize the micro-well with transmitted light through the camera path and choose the ROI symbol. Set the diameter of the ROI to $4,000 \mu \mathrm{m}$ and overlap the edge of the digital ROI to the edge of the current micro-well. Select Lock.

NOTE: the shape and diameter of the ROI depends on the design and size of the PDMS stencil being used. For example, if using $5,000 x$ $5,000 \mu \mathrm{m}$ PDMS squared stencils, use a 5,000 x 5,000 $\mu \mathrm{m}$ squared ROI.

4. Repeat the overlapping step (step 5.3) for each micro-well of the dish. Upon completion, switch off the transmitted light.

5. Navigate to the center of the reference pattern micro-well, away from the reference pattern region, and select PRIMO to upload the desired pattern template, which will be projected on the ROI as a design unit (see Figure 5). Patterns will appear in a dropdown list referred to as Actions and shown in the Actions menu on the software.

NOTE: Pattern templates need to be designed ahead of the experiment and saved as an 8-bit Tiff file before loading the template in the software.

6. In order to create a pattern across the whole micro-well, the design unit needs to be replicated. A design unit covers around $0.1 \mathrm{~mm}^{2}$ of the micro-well area. In the Replication menu, set the desired number of columns and rows (Lines in the software) (see Figure 5).

7. To generate a continuous pattern, adjust the spacing between columns and lines; in this study, patterns of continuous stripes are obtained using -20 to $-35 \mu \mathrm{m}$ spacing (negative spacing) between columns. This negative spacing creates an overlap among design units (Figure $5 B, C)$.

8. Set the laser dose in the Replication menu. The optimal laser dose in this setting and using PLL-PEG is $1390 \mathrm{~mJ} / \mathrm{mm}^{2}$. The patterning duration will be displayed in the Estimated Time panel.

NOTE: In this case laser power is $7.5 \mathrm{~mW} / \mathrm{mm}^{2}$, taking approximately $30 \mathrm{~s}$ to pattern each design unit, using a dose of $1390 \mathrm{~mJ} / \mathrm{mm}^{2}$. For example, a design unit $\left(0.1 \mathrm{~mm}^{2}\right.$ ) replicated in 4 columns and 4 lines (around $1.6 \mathrm{~mm}^{2}$ ), would take 8 min to be patterned. Higher laser doses may be required if the culture dish surface is passivated with PEG-SVA (approximately double laser dose compared to PLL-PEG).

9. Select Lock and wait until the pattern is virtually displayed.

10. To update the parameters of a pattern, click on the related Action, then unlock and update the parameters. Select Lock again when the pattern update is completed.

11. The patterning of multiple proteins in the same micro-well (sequential micro-patterning rounds) requires an accurate alignment of the patterns. To achieve such alignment, upload all sets of desired pattern templates simultaneously (patterns of first and second photopatterning rounds).

12. Set the replication and dose parameters of the pattern templates. After parameters are set, patterns will appear as Actions in the Actions list. Save this template configuration as a file in software (see Figure 5D).

13. On the Actions list, select only the specific actions to be patterned during the first patterning round and deselect the actions that will be patterned during the second patterning round (see Figure 5D).

14. Navigate to the area where the reference pattern was produced in step 4.2 (for example, top region of the reference pattern micro-well, see Figure 4E). Adjust the focus to the optimal Z-position (obtained in step 4.4).

NOTE: It is strongly recommended to select perfect focus system on the microscope being used (if available), which ensures that the optimal Z-position for patterning will be maintained throughout the entire photopatterning process.

15. Select the Play symbol to start patterning. Patterning duration will be displayed in the Estimated Time panel. On Leonardo software version 4.4 patterning is completed when all Actions appear blue in the Visualization menu. 


\section{Protein incubation}

NOTE: Micro-wells are incubated with ECM proteins (preferably fluorescently-labeled). These will only bind to the areas where PEG was cleaved through the photopatterning process described in step 5. Each well contains a PDMS stencil with 4 micro-wells, which will allow testing 4 different conditions simultaneously, for example, incubation of a different protein in each micro-well (see Figure 4D).

1. Use fluorescently-labeled proteins (for example, laminin, fibronectin or fibrinogen conjugated to red or green fluorophores) in order to visualize the micro-patterns (see step 6.7 and 9.4). Alternatively, adsorbed unlabeled proteins can be visualized at later stages using immunofluorescence.

NOTE: ECM proteins (for example, fibronectin) can be labeled using existing protocols ${ }^{33}$ and commercially available fluorescence labeling kits (i.e., Alexa 488 labeling kit), or purchased readily labeled (for example, conjugated fibrinogen-488 or laminin-red fluorescent rhodamine, see Table of Materials).

2. Under sterile conditions, prepare desired concentration of ECM proteins ( $10 \mu \mathrm{g} / \mathrm{mL}$ laminin, fibronectin or fibrinogen in PBS, see Table of Materials and step 4.3).

NOTE: Fluorescently-labeled ECM proteins are light sensitive and should be protected from light (wrap dish in aluminum foil) and should be kept on ice at all times.

3. Under sterile conditions, wash the micro-wells three times with $20 \mu \mathrm{L}$ of PBS to remove the PLPP.

4. Remove $18 \mu \mathrm{L}$ of PBS from all the micro-wells and add $20 \mu \mathrm{L}$ of ECM protein solution to each micro-well. Incubate at room temperature, for 20-30 min and protect from light.

NOTE: Optimal coating incubation times may vary depending on the type and concentration of proteins.

5. After incubation, remove $15 \mu \mathrm{L}$ of ECM protein solution and wash the micro-wells three times with $20 \mu \mathrm{L}$ of PBS. NOTE: Always leave approximately $5 \mu \mathrm{L}$ of PBS in between washes.

6. If patterning with only one protein (one round of micro-patterning), proceed either to storage of the culture dish (step 10) or to cell plating (step 11, and see Figure 2). If performing a second round of micro-patterning with a different protein in the same micro-well, proceed either to storage of the culture dish (step 10) or to blocking unspecific binding sites (step 7, and see Figure 2).

7. Optional quality control step: visualize and image printed patterns using an epifluorescence microscope prior to plating cells. Select appropriate fluorescent channels and adjust exposure times accordingly. NOTE: To compare the fluorescence intensity of patterns between experiments, it is essential to use the same exposure times for the same proteins.

\section{Blocking unspecific binding sites (only for multiple protein patterns)}

NOTE: Micro-patterning with multiple proteins in the same micro-well involves sequential patterning steps (see Figure 2). A blocking agent (PLLPEG or BSA) is added to the micro-wells to prevent cross-binding, which occurs when the second incubated protein (step 9) binds to the first incubated protein (step 6 ), thus avoiding a mixture of proteins within the patterns.

1. Under sterile conditions, add $20 \mu \mathrm{L}$ of PLL-PEG $(0.1 \mathrm{mg} / \mathrm{mL}$ in PBS) or BSA (1\% BSA in PBS) as a blocking step to prevent cross-binding NOTE: Blocking efficiency may vary depending on the nature of proteins being used and the affinity among them. It is recommended to test both PLL-PEG and BSA as blocking agents beforehand (Figure 10D-I).

2. Incubate blocking agent at room temperature for $1 \mathrm{~h}$ and protect from light. Remove $15 \mu \mathrm{L}$ of blocking agent and wash all micro-wells three times with $20 \mu \mathrm{L}$ of PBS. Always leave approximately $5 \mu \mathrm{L}$ of PBS in between washes.

3. Remove $18 \mu \mathrm{L}$ of PBS from all the micro-wells and add $5 \mu \mathrm{L}$ of PLPP to each micro-well, ensuring that PLPP is homogenous on the whole surface of the micro-wells.

\section{Second round of photopatterning (only for multiple protein patterns)}

NOTE: After the first round of photopatterning and protein incubation, a micro-pattern is generated. During the second round of photopatterning, the pattern for the second protein will be generated in the same micro-well (see Figure 2C and Figure 5D). In the software, select the correct pattern templates (actions), which will be patterned during this round (see Figure 5D).

1. Navigate to the first micro-well (Figure 4D). Ensure appropriate overlap between the digital ROI and the micro-well.

2. Load the template configuration saved previously (step 5.12) and select the actions to be patterned during the second photopatterning round (deselect actions from first round, see Figure 5D).

3. Select the Play symbol to start patterning. Ensure the laser is on in the software.

\section{Second round of protein incubation (only for multiple protein patterns)}

NOTE: In this part of the protocol, fluorescently-labeled protein/s will be incubated on the culture dish after the second round of photopatterning.

1. Under sterile conditions, wash the micro-well three times with $20 \mu \mathrm{L}$ of PBS to remove the PLPP. Remove $18 \mu \mathrm{L}$ of PBS and add $20 \mu \mathrm{L}$ of ECM protein solution to each micro-well. Incubate at room temperature for 20-30 min and protect from light.

NOTE: Optimal coating incubation times may vary depending on the type and concentration of proteins.

2. After incubation, remove $15 \mu \mathrm{L}$ of ECM protein solution and wash the micro-wells three times with $20 \mu \mathrm{L}$ of PBS. Always leave approximately $5 \mu \mathrm{L}$ of PBS in between washes.

3. Either proceed to storage of culture dish (step 10) or to cell plating (step 11, and see Figure 2).

4. Optional quality control step: using an epifluorescence microscope, visualize and image printed patterns before plating cells. Select appropriate fluorescent channels and adjust exposure time. 
NOTE: To compare the fluorescence intensity of patterns between experiments, it is essential to use the same exposure times for the same proteins.

\section{Storage of micro-patterns}

NOTE: Micro-patterns with adsorbed proteins can be stored during different steps of the protocol (see Figure 2). If micro-patterning with multiple proteins, micro-patterns can be stored after the first round of micro-patterning or after the two sequential rounds of micro-patterning have been completed (see Figure 2B).

1. When passivated with PLL-PEG, store the micro-patterns in PBS ( $3 \mathrm{~mL}$ per well) at $4{ }^{\circ} \mathrm{C}$ for up to 3 days.

2. If the culture dish was passivated with PEG-SVA, micro-patterns can be stored for up to 1 month. In order to do so, rinse micro-patterns intensively with double distilled deionized water and dry with a sterile air gun of argon or nitrogen, although normal air can also be used. After drying, micro-patterns can be stored at $4{ }^{\circ} \mathrm{C}$ for up to 1 month (PRIMO system support team, personal communication).

\section{Plating cells}

NOTE: During the next steps, cells will be plated on the prepared micro-patterned culture dish/es. In these studies, a neuronal cell line (CAD cells) is used ${ }^{31}$. However this protocol can be adjusted to study other cell types of interest (adjust cell plating protocol as required).

1. Differentiate CAD cells for $48 \mathrm{~h}$ using differentiation medium (DMEM supplemented with $1 \%$ Glutamine, without serum, and with $1 \%$ Pen/ Strep, see Table of Materials).

2. Plate $1 \mathrm{~mL}$ of medium with cells in the inner glass well, covering all micro-wells and place the culture dish in a $37^{\circ} \mathrm{C}, 5 \% \mathrm{CO}_{2}$ incubator.

\section{Representative Results}

Following the above protocol results in micro-patterned surfaces, coated with ECM protein/s of interest. We are using these patterns to track neuronal pathfinding.

Generated patterns should be a precise representation of the template. An example is shown in Figure 6 where a digital pattern template (Figure 6A) representing one design unit (Figure 5B), resulted in defined micro-patterns, ranging from 20 to $2 \mu \mathrm{m}$ width, coated with labeled Fibrinogen (Figure 6B). Using ImageJ, fluorescence intensity measurements were obtained both vertically (Figure 6C) and horizontally (Figure 6E) along the stripe and from a corresponding background region $15 \mu \mathrm{m}$ above each stripe. The background measurements were subtracted from the pattern measurements for each stripe width.

One limitation of the system is that an edge effect can be observed (Figure 6B, top stripe) when printing features $\geq 20 \mu \mathrm{m}$, with a higher intensity signal at the pattern edges compared to the center (Figure 6D, first peak of fluorescent intensity profile). In our experiments the resolution limit was approximately $2 \mu \mathrm{m}$; at this width we observed a significant decrease (by approximately $50 \%$ ) in fibrinogen fluorescence intensity compared to the intensity of the wider stripes (Figure 6F,G). Patterning using the PRIMO system and the protocol outlined here produced reproducible patterns, with the highest standard deviation of the mean fluorescent intensity measured for the $2 \mu \mathrm{m}$ width from four individual replicated design units (Figure 6G). Variation within the patterned stripes was also found to be low; the coefficient of variation ranged from 3 to $10 \%$, with the 20 $\mu \mathrm{m}$ and $2 \mu \mathrm{m}$ stripes having the largest internal variation. This is likely to be a result of the edge effect and the resolution limit of the system, respectively. Note that for these measurements we only measured the intensity at the center of the stripes, to avoid the uneven illumination resulting from the objective used to acquire these images (vignetting, Figure 6E).

Certain experiments may involve questions that require defined protein concentrations, which can be achieved in two ways: 1) varying the protein concentration (Figure 7A,B). Incubation with different concentrations of laminin, results in significantly different fluorescence intensities, increasing with higher protein concentrations (Figure 7B). 2) The laser dose that is used to cleave the anti-adhesive film (PEG) can be varied. Higher laser doses will remove the antifouling film to a greater extent, generating more binding sites for the proteins of interest (Figure 7C,D) resulting in significantly different fluorescence intensities, increasing with higher laser doses (Figure 7D).

Varying the laser dose allows the generation of protein gradients within the same pattern. This is displayed in Figure $\mathbf{8 A}$, where a gradient template was designed using different greyscale levels, from black (no laser power) to white (maximum laser power).

Laser intensity is proportional to the greyscale level of the template (ranging from 0 to 255 in an 8-bit image), generating gradients of UV illumination. The measurement of the fluorescence intensity profile along the gradient stripe is linear in the pattern template (Figure 8B) and in the generated gradient pattern (Figure $\mathbf{8 C}, \mathbf{D}$ ). This is reproducible among all gradient stripes within the same template and gradient pattern (Figure 8B,D). Generation of such gradients is extremely useful and helps to mimic in vivo environments where cells often respond to gradients of bioactive proteins ${ }^{34,35,36,37}$.

Cells sense changing extracellular environments but assays that enable the study of cell behavior when cells encounter such changes are limited. LIMAP can be used to micro-pattern with multiple proteins in the same micro-well. Examples are shown in Figure 9 where cross-patterns were generated with stripes of fibronectin (horizontal) and laminin (vertical). When creating patterns with multiple proteins, it is crucial to use a blocking step between the first and second protein incubation, to prevent cross-binding of proteins (see step 7). The blocking efficiency may vary depending on the biochemical characteristics of the proteins that are used for coating and we advise testing several blocking buffers including PLL-PEG $(0.1 \mathrm{mg} / \mathrm{mL})$ and BSA (1\%). To evaluate this cross-binding effect, we performed fluorescence intensity measurements using Image J (Figure 9) and we showed that cross-binding can be reduced dramatically, using PLL-PEG buffer $(0.1 \mathrm{mg} / \mathrm{mL})$ for fibronectin and laminin crosspatterns (Figure 9D,H). 
The generated cross-patterns were used for cellular assays with CAD cells (Figure 10A,B) or rat dorsal-root ganglion (DRG) neurons (Figure 10C). Their neurites (CAD) and axons (DRG) grow along different lines. CAD cells are used as a neuronal model since they show a similar integrin expression profile compared to primary neurons and they still display actin-rich growth cones after $48 \mathrm{~h}$ in culture (Figure 10B), making them suitable for pathfinding studies.

In order to investigate the possible cytotoxic effects of the generated micro-patterns towards primary neurons, DRG neurons were isolated and cultured on micro-patterns following a previously published protocol ${ }^{38}$. The results demonstrate that primary neurons tolerate the micro-patterns environment (Figure 10C). We are currently studying how a variety of ECM proteins influence axonal (neurite) pathfinding. Preliminary proof of concepts found in CAD cells will be further investigated using DRG neurons. In order to validate the quality of generated micro-patterns, it is desirable to image patterns by fluorescence microscopy to ensure the pattern edges are well-defined before proceeding to cell plating. During the imaging process, it is important to ensure the optical adjustment between the microscope and the camera to avoid the peripheral darkening effect (vignetting) which affects the posterior analysis and interpretation of data. Additionally, acquire an image of a pattern-free region using the same exposure times that will be used to image the patterns and subtract this image from the pattern image.

In summary, for good quality micro-pattern generation, it is advisable to assess the protein concentration (Figure 7A,B), laser doses (Figure 7C,D), protein background levels (Figure 6E,F,H) and an efficient blocking step (Figure 9) when using multiple proteins. Conclusively, the quality of the micro-patterns generated with LIMAP is essential in order to obtain reliable and reproducible data from cellular assays.

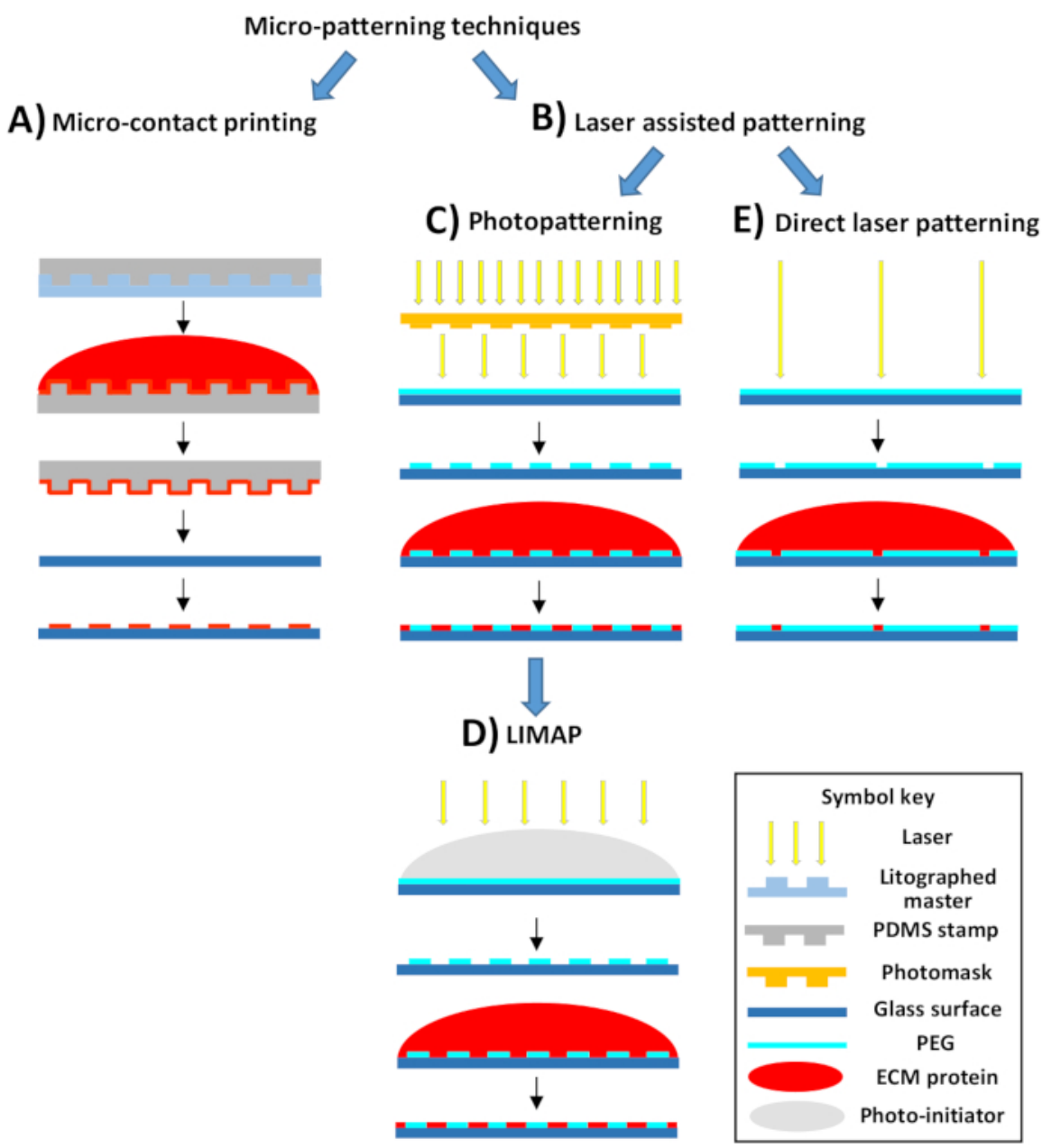

Figure 1: Scheme of micro-patterning techniques: microcontact printing and laser-assisted patterning. (A) Microcontact printing uses a lithographed master with defined micro-features to generate a PDMS stamp which is incubated with the protein of interest. This protein is then transferred (stamped) onto a glass surface, generating protein micro-patterns. (B) Laser-assisted patterning techniques include photopatterning and direct laser patterning. (C) Most photopatterning approaches use a UV light source and a photomask (either in contact with the substrate surface or in the focal plane of the objective) with desired geometries in order to cleave the PEG antifouling surface in specific positions, creating a defined pattern. A subsequent protein incubation step results in protein adsorption only to the laser-cleaved regions. (D) LIMAP is a photopatterning technique which does not require a photomask in contact with the substrate (i.e., a maskless and contactless approach). LIMAP uses a photo-initiator, which is activated by low doses of a laser, cleaving light-exposed regions of PEG. This creates attachment sites for sequential protein adsorption. (E) Direct laser patterning uses high energy light to directly etch the PEG film, allowing protein binding in those etched regions. Please click here to view a larger version of this figure. 
A

Micro-patterning with one protein (one round of micro-patterning)

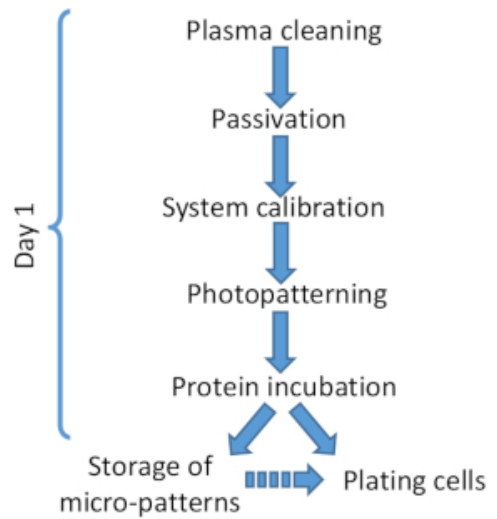

C

Micro-patterning

with one protein

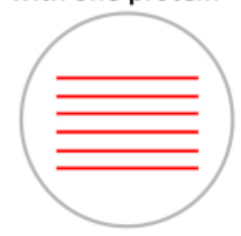

Micro-patterning with multiple proteins

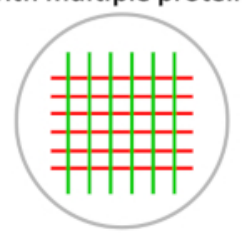

B

Micro-patterning with multiple proteins

in the same micro-well

(two sequential rounds of micro-patterning)

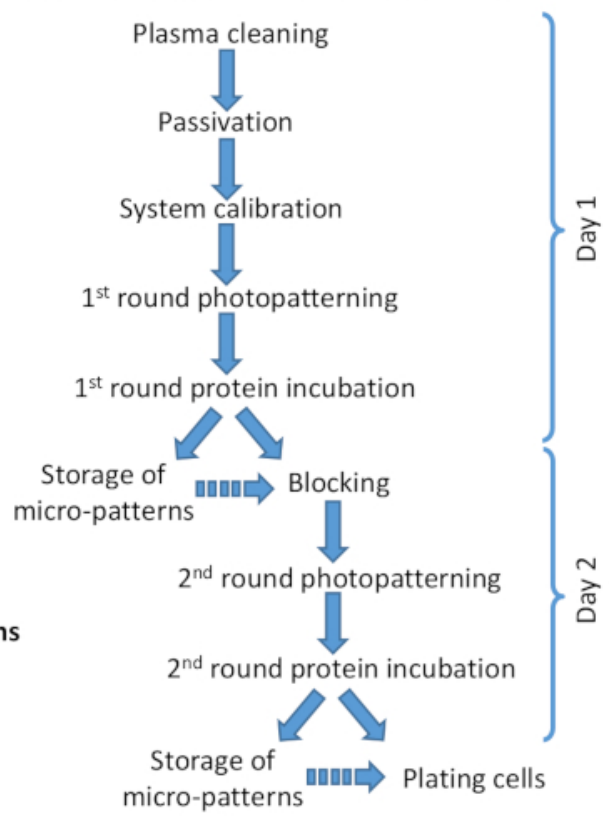

Figure 2: Scheme showing a summary of the steps in the micro-patterning protocol. (A) Micro-patterning with one protein involves only one round of micro-patterning (photopatterning and protein incubation) and can be performed in under $8 \mathrm{~h}$. (B) Micro-patterning with multiple proteins requires two sequential rounds of micro-patterning and can be completed in 1-2 days, depending on the number of micro-patterns being prepared. It is possible to go through the $B$ version of the protocol in 1 day of work. Continuous arrows indicate direct flow of steps in the protocol. Discontinuous arrows indicate that there is a significant time gap between one step and the other (see step 6.6 and 9.3). (C) Schematic view of example patterns obtained after one round of micro-patterning (red stripes) or two sequential rounds of micro patterning (red and green stripes). Please click here to view a larger version of this figure. 


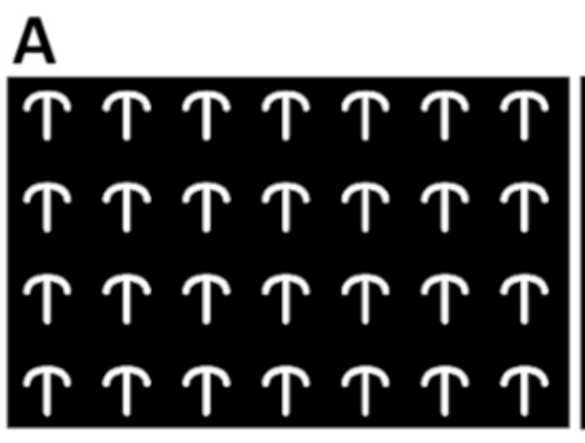

C

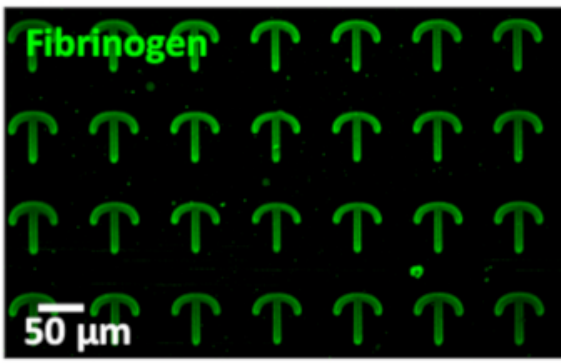

B

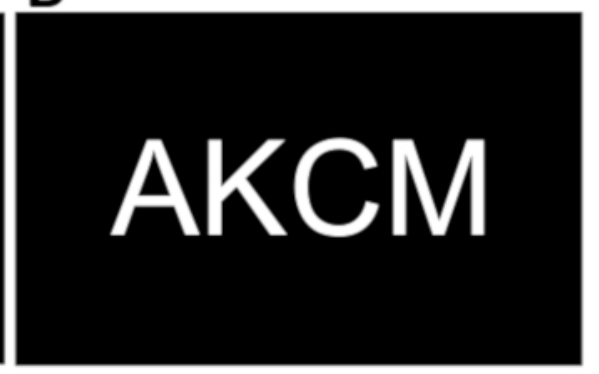

D

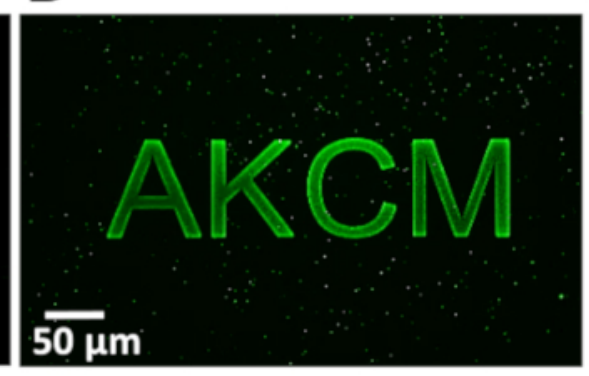

Figure 3: Pattern template generation is versatile with LIMAP. (A,B) Examples of pattern templates designed with ImageJ (A crossbows, $B$ letters). Shapes drawn in white were projected at maximum laser power and shapes drawn in black were not projected. (C,D) Micro-patterns obtained with LIMAP from templates after incubation with $10 \mu \mathrm{g} / \mathrm{mL}$ fibrinogen (green). (C) Crossbows are $50 \mu \mathrm{m}$ width and $50 \mu \mathrm{m}$ height spaced by $75 \mu \mathrm{m}$ horizontally and $50 \mu \mathrm{m}$ vertically. (D) Letters are $80 \mu \mathrm{m}$ width and $85 \mu \mathrm{m}$ height. Scale bars in C and D represents $50 \mu \mathrm{m}$. Please click here to view a larger version of this figure. 
A

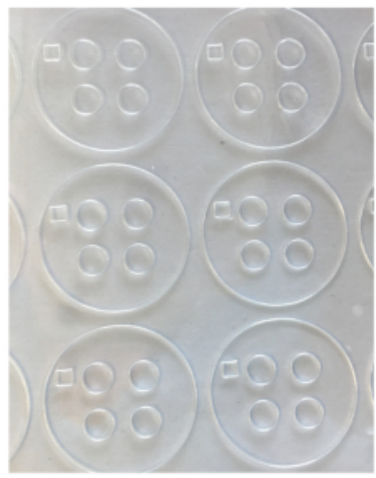

C

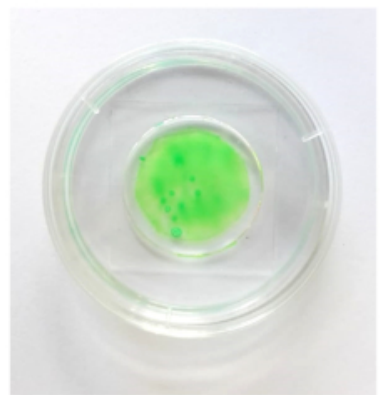

B

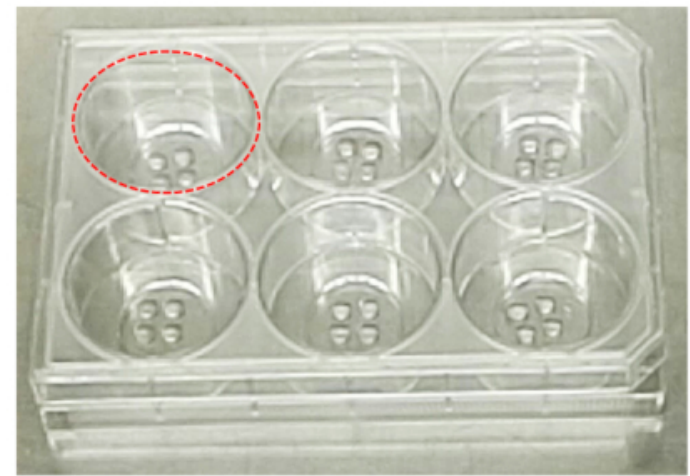

D

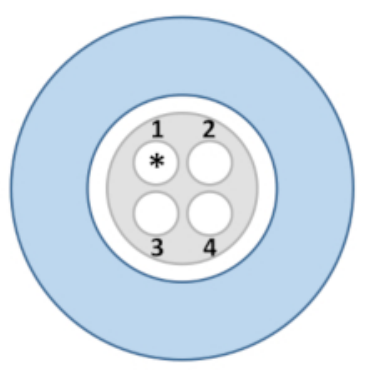

E

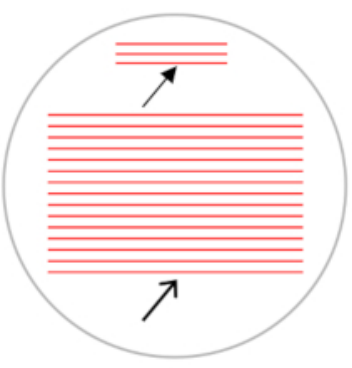

Figure 4: Essential materials for the LIMAP protocol. (A) Stencils used in this protocol are $20 \mathrm{~mm}$ diameter, thin circular-shape PDMS pieces ( $250 \mu \mathrm{m}$ thickness) containing 4 micro-wells ( $4 \mathrm{~mm}$ diameter each). The volumes used in the micro-wells range from 5 to $20 \mu \mathrm{L}$, considerably reducing the amount of reagents and proteins needed for each experiment. (B) 6 well glass bottom dish where stencils have been already placed in each well. Micro-wells contain $20 \mu \mathrm{L}$ of PBS to make them visible. (C) Calibration dish in which the inner glass well has been marked with a green highlighter, which will be used to calibrate the laser focus. (D) Schematic view of top left well from the 6-well glass bottom dish in $B$ (outlined with dashed red circle). The inner glass bottom well is represented in white and the stencil is shown in grey. The stencil contains 4 micro-wells (numbered 1-4), for the testing of 4 different experimental conditions (e.g., different protein concentrations, pattern geometries, combinations of proteins, etc.). The asterisk represents the micro-well containing the reference pattern. (E) Schematic view of micro-well where a reference pattern has been generated in the top part (arrow with filled arrowhead). This reference pattern is required to obtain the optimal laser focus for patterning (see step 4). Arrow with empty arrowhead indicates the central area of the micro-well, which will be used for subsequent patterning after system calibration. Please click here to view a larger version of this figure. 

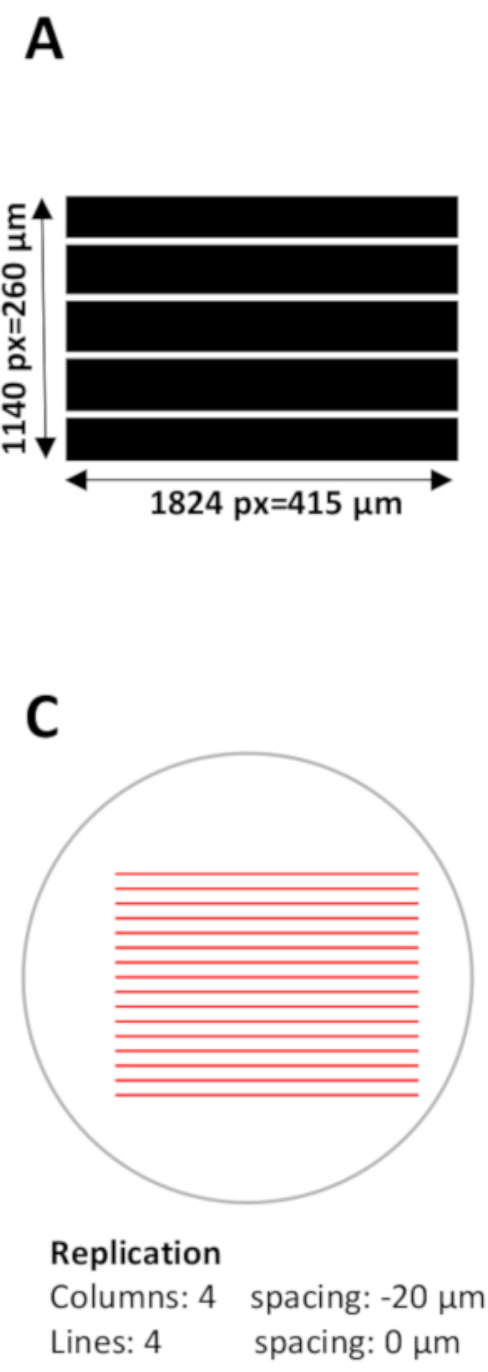

B

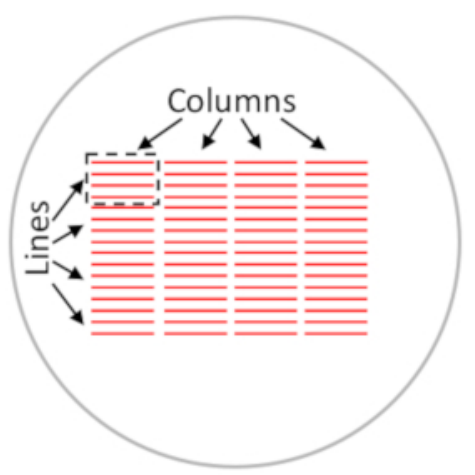

\section{Replication}

Columns: 4 spacing: $0 \mu \mathrm{m}$

Lines: 4 spacing: $0 \mu \mathrm{m}$

D

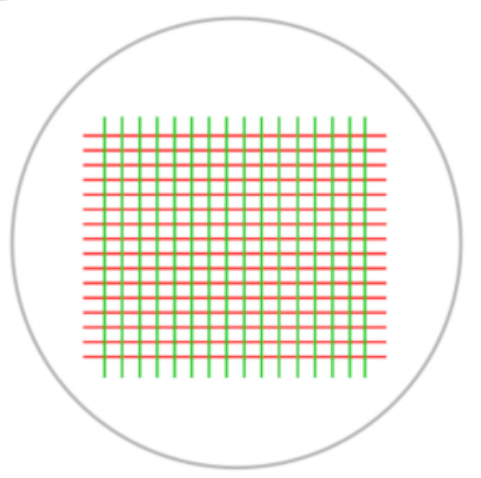

Actions list

$1^{\text {st }}$ round of micro-patterning

$\square$ Action 1

$\square$ Action 2

\section{$2^{\text {nd }}$ round of micro-patterning}

$\square$ Action 1

$\checkmark$ Action 2

Figure 5: Software set-up for micro-patterning. (A) Pattern template with parallel stripes designed with ImageJ and saved as an 8-bit Tiff file. (B-D) Schematic view of digital ROls (regions of interest) that will overlap with the current micro-wells where micro-patterns will be generated. (B) The pattern template to be used (length 1824 pixel=415 $\mu \mathrm{m}$, width 1140 pixel=260 $\mu \mathrm{m}$ ) is selected on Leonardo and is projected on the ROI as a design unit (red stripes in the black dashed rectangle), which will cover approximately $0.1 \mathrm{~mm}^{2}$ of the micro-well area. The design unit is replicated in 4 columns and 4 lines in the Replication menu (template configuration), creating a pattern across the micro-well. NOTE the space among the columns. (C) To pattern continuous stripes, the spacing between columns has to be adjusted. In this case, to achieve an overlap among design units the spacing between columns is set in the replication menu as Negative spacing, $-20 \mu \mathrm{m}$. (D) In order to pattern multiple proteins in the same micro-well, an accurate alignment of the patterns is required. During the software set-up step (step 5), upload all desired pattern templates simultaneously. On the Actions list, select only the specific actions to be patterned during each patterning round and deselect the rest of the actions (step 5.12, 5.13 and 8.2). Please click here to view a larger version of this figure. 
A

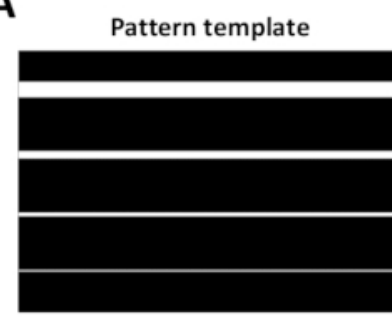

C

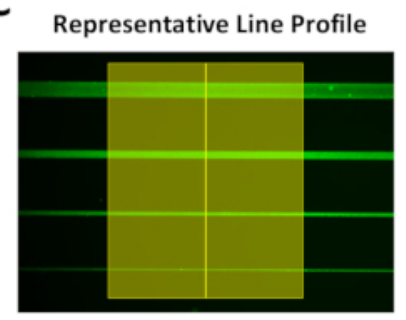

E

Representative Line Profiles

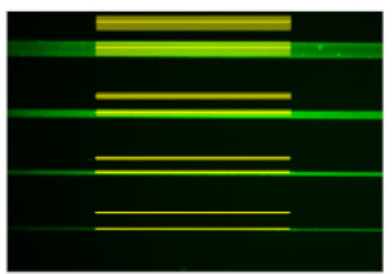

G

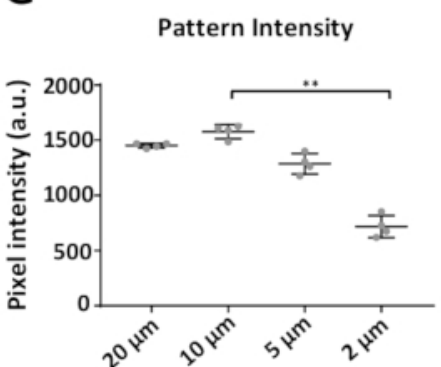

B

Generated micro-pattern

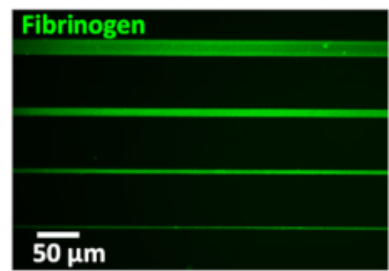

D

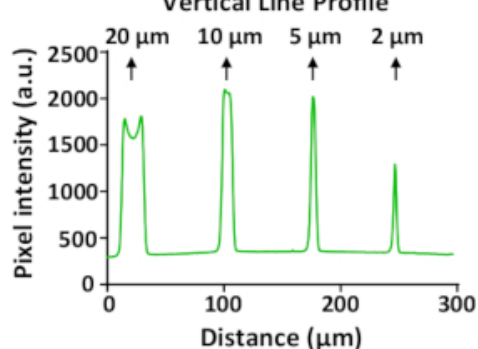

F

Horizontal Line Profiles

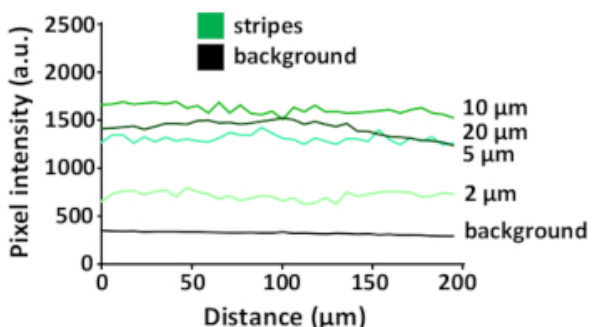

$\mathrm{H}$

Stripe Intra-variation

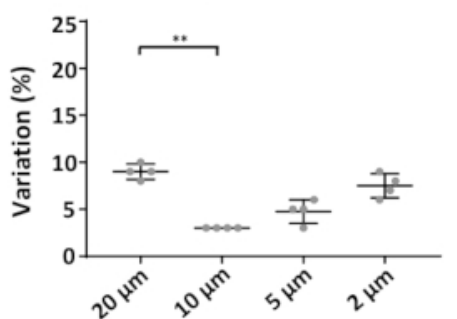

Figure 6: Analysis of pattern variability using LIMAP. (A) Pattern template designed with ImageJ used to micro-pattern four stripes of varying width $(20,10,5,2 \mu \mathrm{m}$, from top to bottom). (B) Micro-pattern obtained after incubation with $10 \mu \mathrm{g} / \mathrm{mL}$ of fluorescently-labeled fibrinogen (green). (C) Intensity measurements along a vertical line crossing the stripes of the micro-pattern. (D) Vertical fluorescence intensity profile obtained from measurement in (C). NOTE that at larger widths $(20 \mu \mathrm{m})$ there is a variation in the vertical profile caused by the accumulation of protein at the edges of the stripe, resulting in two distinct fluorescence intensity peaks (edge effect). This effect is only seen in stripe widths $\geq 20 \mu m$. (E) Intensity measurements along the depicted horizontal lines (fluorescence and background). (F) Horizontal fluorescence intensity profiles obtained from measurements in (E). (G) Graph showing the mean intensity for each stripe width, measured from four individual replicated design units (inter-pattern variation). NOTE the reduced protein adsorption to patterns of $2 \mu \mathrm{m}$ stripe width. $(\mathbf{H})$ The variation within the patterned stripes (coefficient of variation) was low for all stripe widths, ranging from 3 to $10 \%$. Data in $G$ and $H$ shown as mean \pm SD. Statistical analysis in $G$ and $\mathrm{H}$ was performed using one-way ANOVA (Kruskal-Wallis) non-parametric test with multiple comparisons. $\mathrm{P}$ value is $<0.001 \mathrm{for}$ ** significance. Please click here to view a larger version of this figure. 
A

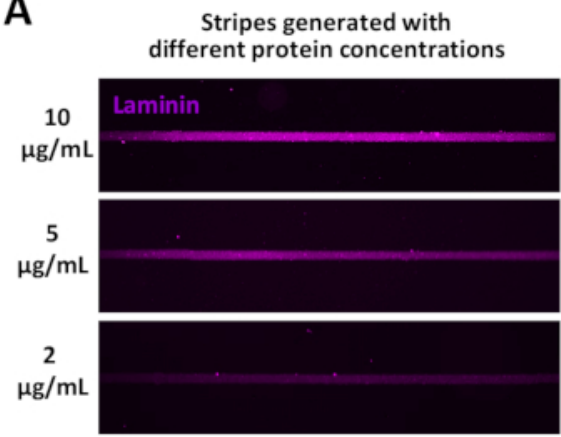

C

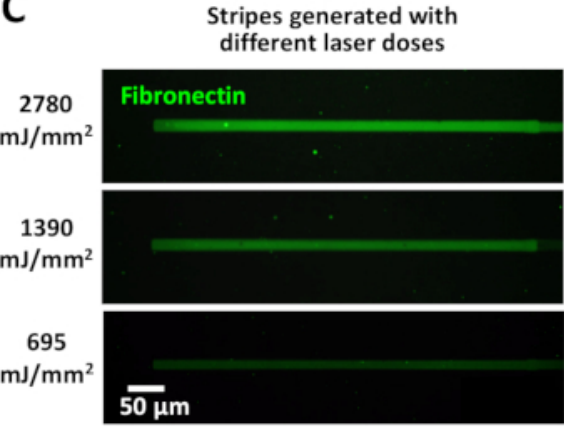

B

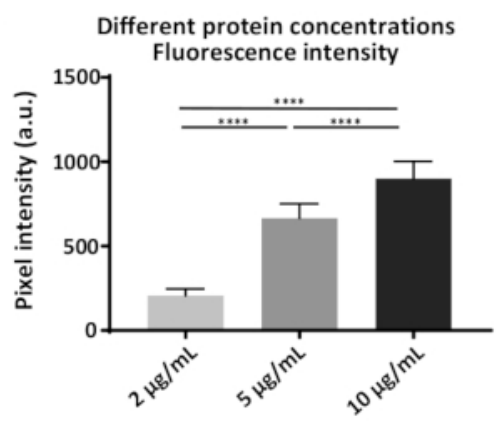

D

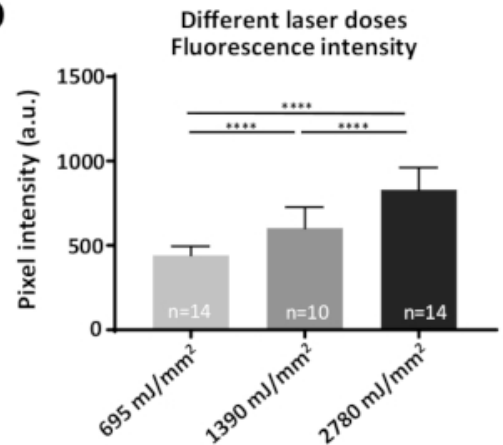

Figure 7: The effect of variations in laser power and protein concentration for protein adsorption efficiency. (A) The PLL-PEG surface was laser-cleaved with a constant laser dose $\left(1390 \mathrm{~mJ} / \mathrm{mm}^{2}\right)$ and incubated with the indicated concentrations of fluorescently-labeled laminin (magenta). (B) Quantification of fluorescence intensity of the laminin stripes in (A). (C) The different indicated laser doses were applied followed by incubation with the same concentration $(10 \mu \mathrm{g} / \mathrm{mL})$ of fluorescently-labeled fibronectin (green). (D) Quantification of fluorescence intensity of the fibronectin stripes in (C) showing that higher laser doses correlate to higher levels of adsorbed protein. All measurements are background subtracted. Sample numbers are indicated at the bottom of columns; data is shown as mean \pm SEM. Statistical analysis was performed using non-parametric Mann-Whitney test with two-tailed calculation. P-value is $<0.0001$ for ${ }^{* * *}$ significance. Please click here to view a larger version of this figure. 
A

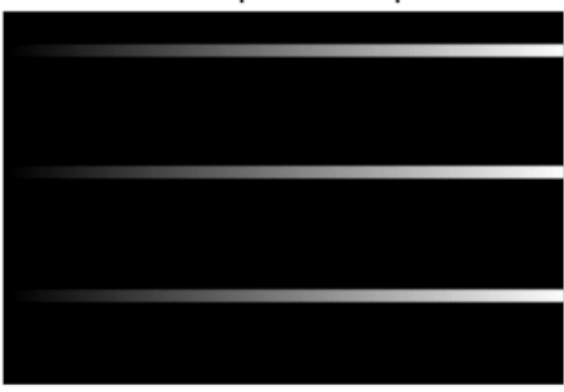

C Generated pattern gradient

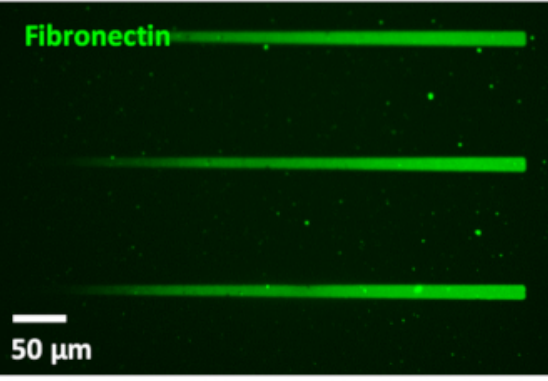

B

Template intensity profile

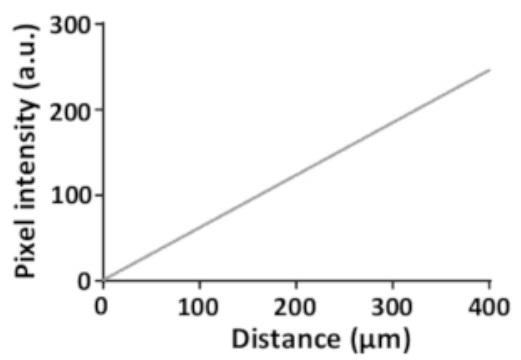

D

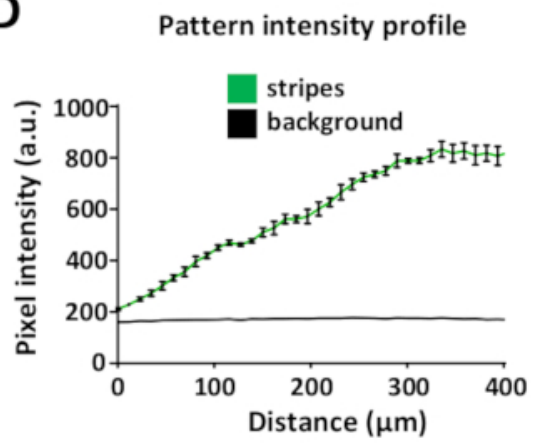

Figure 8: Generation of a protein concentration gradient within a micro-pattern. (A) Gradient pattern template in greyscale. (B) Fluorescence intensity profile measured from (A). (C) Pattern obtained with LIMAP from pattern template in $(A)$ after incubation with $10 \mu g / m L$ of fluorescently-labeled fibronectin (green). (D) Fluorescence intensity profile of $n=3$ stripes and background represented as mean \pm SEM, showing the linear increase in intensity of the protein gradient. Please click here to view a larger version of this figure. 

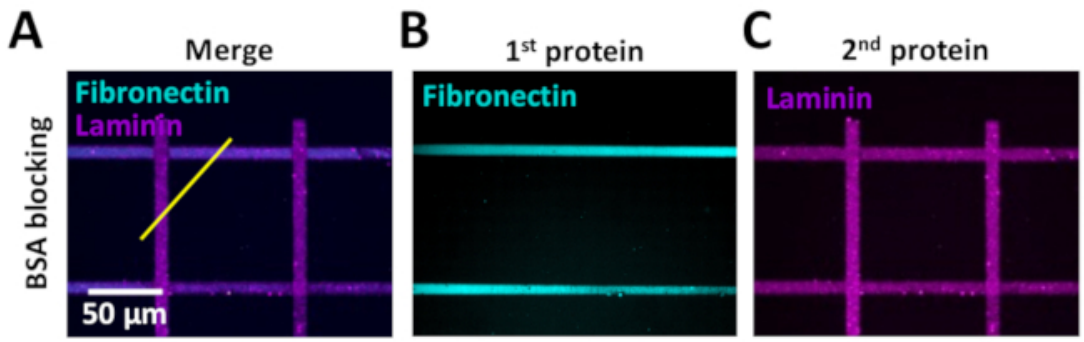

$$
\begin{gathered}
\text { Cross-pattern intensity } \\
\text { BSA blocking }
\end{gathered}
$$
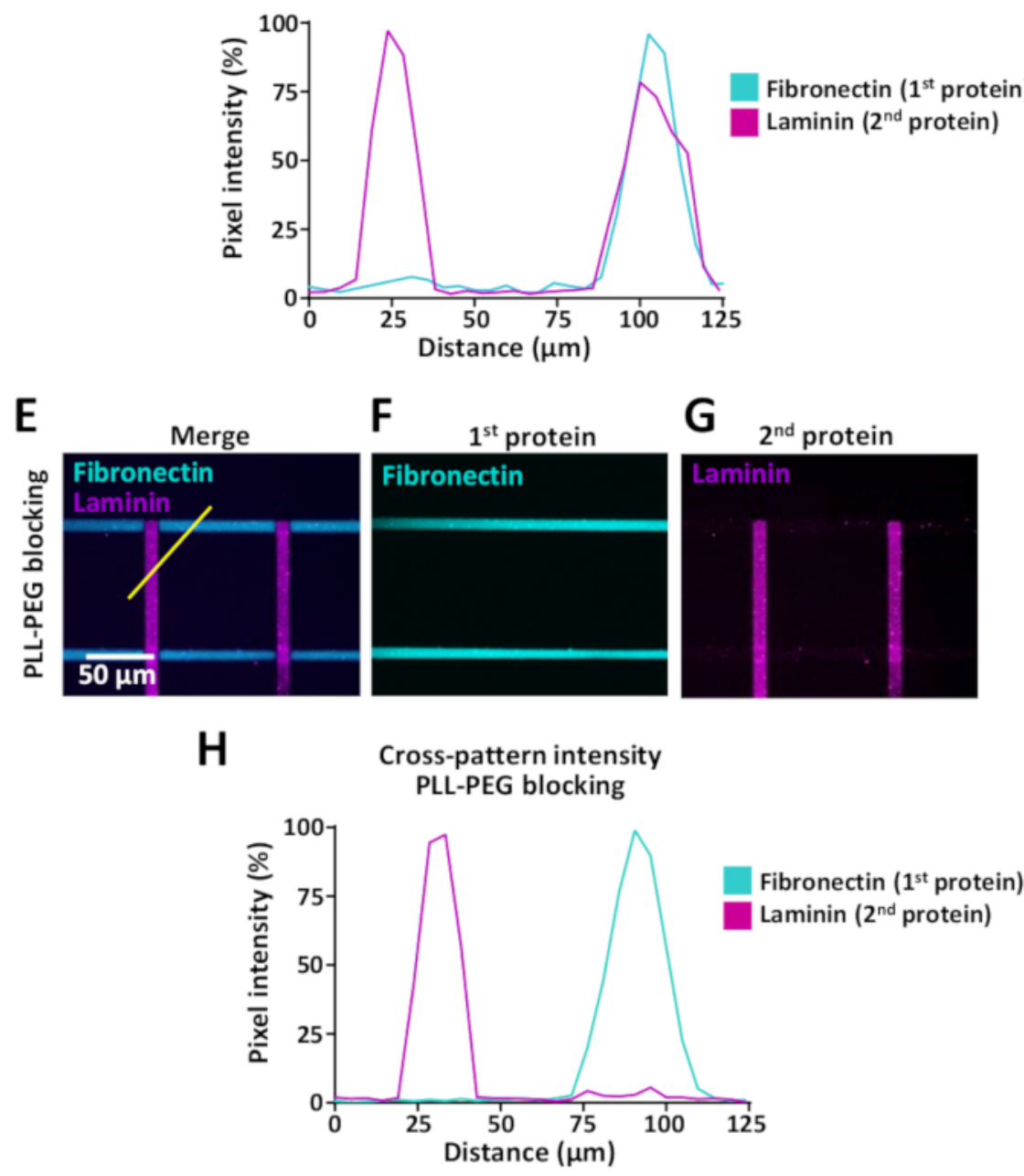

Figure 9: The cross-binding effect when patterning several proteins sequentially. (A-C, E-G) Cross-patterns with $10 \mu \mathrm{m}$ stripes of fluorescently-labeled fibronectin (cyan, horizontal) and fluorescently-labeled laminin (magenta, vertical). (A-C) Samples treated with BSA blocking buffer. (E-F) Samples treated with PLL-PEG for blocking unspecific binding sites (step 7). (A,E) Merged fluorescence channels showing both fibronectin and laminin. (B,F) Image showing fibronectin only. $(\mathbf{C}, \mathbf{G})$ Image showing laminin only. For $C$, note the presence of laminin also on horizontal fibronectin positive stripes which is due to the ineffective blocking of unoccupied binding sites with BSA. For G, note that blocking with PLL-PEG prevents efficiently binding of laminin to the fibronectin stripes. (D,H) Fluorescence intensity profiles obtained from indicated measurements (diagonal yellow line) in $\mathrm{A}$ and $\mathrm{E}$, respectively. Please click here to view a larger version of this figure. 
A

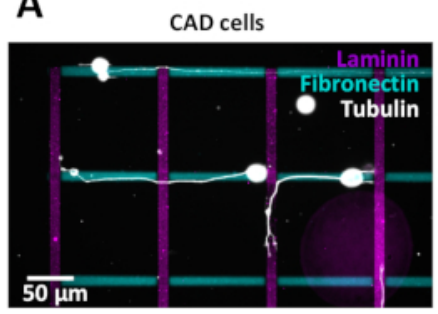

B Growth cone of

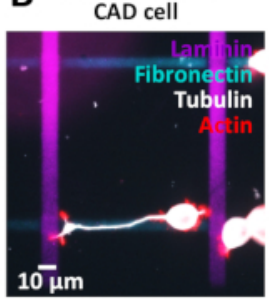

C

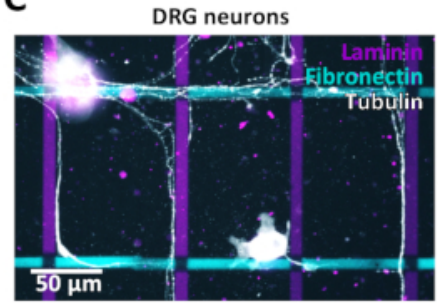

Figure 10: Cross-patterns to investigate neurite/axon pathfinding. (A-C) Cross-patterns with $10 \mu \mathrm{m}$ stripes of fluorescently-labeled fibronectin (cyan, horizontal) and fluorescently-labeled laminin (magenta, vertical). (A,B) Fluorescent images of CAD cells with neurites growing along the micro-patterns. To visualize neurites, cells were cultured for $48 \mathrm{~h}$, fixed with $4 \%$ PFA and stained for tubulin (A) or tubulin and actin (B). (C) Rat dorsal-root ganglion (DRG) neurons with axons growing along the micro-patterns. To visualize axons, DRG neurons were cultured for 72 $\mathrm{h}$, fixed with $4 \%$ PFA and stained for tubulin. Please click here to view a larger version of this figure.
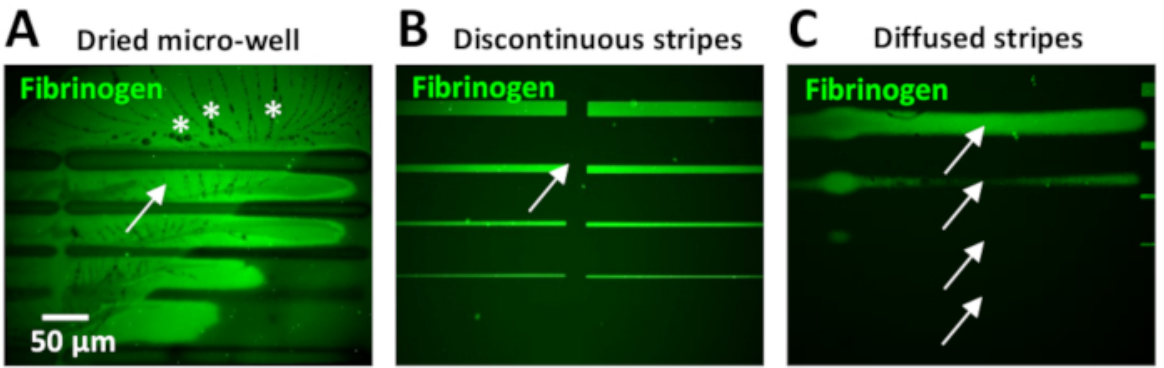

Figure 11: Examples of common negative results obtained when generating micro-patterns with LIMAP. (A-C) Sub-optimal patterned stripes of $10 \mu \mathrm{g} / \mathrm{mL}$ fluorescently-labeled fibrinogen (green) obtained under different circumstances. (A) The micro-well dried out during pattern generation. Note the high levels of fluorescence in the background (arrow) and the presence of PBS crystals (asterisks). (B) The stitching between the stripes was not properly adjusted during software set-up resulting in discontinuous stripes with gaps (arrow) among design units (see step 3.4.7). (C) The laser focus was sub-optimal causing diffused stripes (arrows) which do not represent the actual stripe widths of the pattern template, which should be 20,10,5, $2 \mu \mathrm{m}$ from top to bottom, as in (B). Please click here to view a larger version of this figure.

\section{Discussion}

\section{LIMAP (PRIMO) micro-patterning advantages and comparison with microcontact printing}

While microcontact printing is possibly the most commonly used micro-patterning technique in the biological field ${ }^{39}$, there seems to be an increasing number of researchers using LIMAP technology $40,41,42,43,44$. Here, we presented a protocol using PRIMO, a commercially available system for LIMAP. Below we briefly discuss potential advantages and limitations of microcontact printing and LIMAP photo-patterning.

Microcontact printing requires lithographed masters produced by spin-coating a photo-mask (generally SU-8) onto a glass or silicon wafer, which is then laser-etched with the desired micro-features. These masters are used as templates to create a PDMS stamp ${ }^{45}$. The stamp is incubated with a chosen protein that adsorbs to it, and is then transferred (stamped) onto the cell culture dish. The process of adsorption of the protein to the PDMS stamp is dependent on protein concentration, buffer and incubation time. These parameters need to be tested beforehand for optimal results ${ }^{46}$.

Masters can be used in a substantial number of experiments, lasting for months or even years, if correctly preserved. However, a limiting factor of this technology is the necessity to re-design new lithographed masters for every desired modification. Changes in experimental designs may result in time-consuming production of new masters (up to several weeks) thus delaying experiments. In comparison, LIMAP photopatterning does not require a physical master; it uses software-generated pattern templates that can be used to flexibly adapt desired geometries of micropatterns to changing research questions. LIMAP can be also used to generate protein gradients within the same micro-pattern (Figure 8), which is harder to obtain in a reproducible manner using microcontact printing ${ }^{47}$.

Furthermore, the micro-pattern resolution achieved with LIMAP, in our case, is $2 \mu \mathrm{m}$ (Figure 6B).

Approaching this resolution increased intra- and inter- pattern variability. Generating patterns around or above $10 \mu \mathrm{m}$ width was highly reproducible (Figure $\mathbf{6 G}, \mathbf{H}$ ). On the contrary, with microcontact printing it is difficult to consistently obtain resolutions below $10 \mu \mathrm{m}$ and it is common to find artefacts when stamping small features (data not shown).

We have shown that LIMAP can be used to micro-pattern multiple proteins (Figure 9) within the same micro-well, allowing further levels of complexity to be added to experiments. Although this could be achieved with microcontact printing, aligning different proteins with high level of precision can be technically rather demanding. Whilst patterning multiple proteins using LIMAP seems straight forward, it is important to mention that cross-binding of proteins through sequential coating procedures can be reduced through blocking reagents but not entirely eliminated (Figure 9).

Regarding the cost of one or the other technique, LIMAP as described here requires the purchase of micro-patterning equipment (PRIMO) that can be installed on different fluorescence microscopes and requires a motorized stage. Although this investment is initially cost intensive, there 
are no additional purchases other than consumable items (stencils, PEG and PLPP) on the long run associated with LIMAP. Alternatively, the PDMS stencils can also be produced in the lab by the own experimenter following published protocols ${ }^{18,32}$. The largest costs for microcontact printing may be associated with the production of new masters, which can become substantial if experiments require new patterns.

One drawback of LIMAP is the relatively low throughput approach of this technique. Microcontact printing can produce a large number of micro-patterns quickly and efficiently in a simultaneous stamping step, compared to the required sequential laser micro-patterning with LIMAP. For example, it is possible to produce 6 stamped glass coverslips in about $2 \mathrm{~h}$ with microcontact printing using PDMS stamps (excluding stamp preparation); patterning a similar area (6-well dish) with LIMAP would take around $4 \mathrm{~h}$, excluding the procedure of surface passivation (considering the pattern template configuration described in step 5.12 and see Figure 5B).

Another rate limiting factor of LIMAP technology is the long illumination time required for patterning large areas $(30 \mathrm{~s}$ per design unit with a $7.5 \mathrm{~mW} / \mathrm{mm}^{2}$ laser). In these cases, microcontact printing might be a preferred option. A newly available photo-initiator (PLPP gel, Table of Materials) should considerably reduce the time taken for the patterning, allowing the generation of hundreds of micro-patterns in large areas (up to $8 \mathrm{~mm}^{2}$ ) in just a few minutes.

Another important factor to take into account when micro-patterning surfaces for cell culture is the reproducibility of the micro-patterns among different experimental repeats, in comparison to the variability obtained with microcontact printing. For example, the graphs shown in Figure 7B,D are representative data of three independent experimental repeats with very similar results (data not shown). Based on our experience and previous publications, this level of reproducibility is difficult to achieve with microcontact printing $48,49,50,51,52$.

In contrast to other photo-patterning techniques that require either dedicated chemistry to engineer photosensitive materials or the use of photosensitizers, which are generally not very biocompatible ${ }^{3}$, the photosensitive component of LIMAP (PLPP) is biocompatible and well-tolerated by cells ${ }^{21}$; in our hands we have not experienced any cytotoxicity across a variety of cells, including CAD, DRG neurons (Figure 10), fibroblasts, epithelial cells, and melanoma cells (data not shown). Another advantage of LIMAP using PRIMO compared to other photo-patterning techniques is that no photomask is required. Similar to microcontact printing, new photomasks would need to be designed and generated for every desired pattern.

All the limitations mentioned above for microcontact printing, refer to the manual approach of the technique. However, it is possible to enhance the throughput and the reproducibility of microcontact printing using an automated device with stamp load and pressure control ${ }^{53}$.

\section{Key steps of the protocol and problems solving for LIMAP using PRIMO}

One of the most common problems found during this protocol is having high levels of background fluorescence within the micro-patterns. This can be due to the drying out of micro-wells which often occurs due to their small volume. When this occurs, PBS crystals often appear surrounding the ECM patterns (Figure 11A).

Insufficient or inefficient washing steps after protein incubation can also result in high levels of background fluorescence. This can be observed particularly upon using protein concentrations of $10 \mu \mathrm{g} / \mathrm{mL}$ (Figure 11B) or higher. The excess of protein in the background can be reduced by including additional washing steps with PBS.

The presence of protein background needs to be measured and characterized in each experiment, calculating the background fluorescence intensity (Figure 6E) and subtracting it from the micro-patterns intensity (Figure 6F-H and Figure 7B,D). High protein background may have an impact in the attachment and sprouting of CAD cells, compromising the interpretation of results.

Having gaps between design units is a common problem when users have limited experience (Figure 11B), which occurs as a result of insufficient overlap between patterns. Two parameters in the Leonardo software can be adjusted to overcome this: 1) a negative spacing between columns may be required, depending on the design of the pattern (step 5.7 and see Figure 5B,C). Alternatively, 2) use the gradient option in the Expert menu to stitch the columns. A quick test to determine the optimal spacing parameters can be performed using UV adhesive (Table of Materials). A small drop of this adhesive is applied to a glass slide, which is then covered with a glass coverslip, making a film. The embedded UV adhesive is photopatterned with the pattern template of interest using a low laser dose $\left(30 \mathrm{~mJ} / \mathrm{mm}^{2}\right)$. The UV- exposed regions of the embedded adhesive will be cured, becoming visible under bright-field microscopy. The test results are visualized to evaluate the obtained spacing within the pattern. In our neuronal experiments, a gap between stripes may adversely affect cell behavior, producing variations in growth dynamics (either reduced speed or abandonment of the path).

In the latest update of Leonardo software (at the time of publication, Leonardo 4.11), it is possible to upload previously designed bigger pattern templates that cover a much larger area (up to $8 \mathrm{~mm}^{2}$ using the $20 X$ objective) of the micro-well surface compared to the current $0.1 \mathrm{~mm} \mathrm{~m}^{2}$ per design unit, eliminating the need to stitch together the smaller design units. Undefined edges can result from a lack of laser focus adjustment during pattern generation (Figure 11C). It is therefore critical to calibrate the laser and perform reference pattern steps (see step 4) prior to patterning. Poorly defined stripes result in variations in stripe width, making the correlation between axon growth dynamics and stripe width difficult. Axons also tend to abandon stripes that have diffused edges. Additionally, variability in edges can also be found when printing stripes of $10-20 \mu \mathrm{m}$ width or higher, resulting in a higher protein content at the edges compared to the central regions of the pattern (Figure 6B,D). This edge effect is produced by a non-homogeneous diffusion of the photo-initiator during the photopatterning process. The photoscission reaction is oxygen dependent, which diffuses more at the edges. This edge effect can be minimized homogenizing the photo-initiator with a pipette in the micro-well during the photopatterning process. Furthermore, a new commercialized photo-initiator (PLPP gel), can also reduce the edge effect (PRIMO system support team, personal communication).

Micro-printing of more than one protein can result in cross-binding (Figure 9A-D). This can be minimized by increasing the blocking efficiency that is used to occupy unspecific binding sites between the incubation steps for the two different proteins. Cross-binding of proteins can perturb reproducibility of experimental outcomes and may lead to misinterpretation of data, since it is difficult to determine the contribution of each protein to axon growth dynamics and to other cell behaviors.

\section{Conclusion}


We hope that the provided protocol using LIMAP facilitates the generation of protein micro-patterns through the use of the PRIMO system. Whilst our protocol focuses on how to reliably produce micro-patterns in 2D glass surfaces, others have shown that it is possible to use LIMAP for micro-patterning of soft substrates ${ }^{54}$, and microstructured surfaces for $3 \mathrm{D}$ cultures ${ }^{42}$. These micro-patterns can be a versatile tool to study cellular responses to changes in their micro-environment.

\section{Disclosures}

The authors have nothing to disclose.

\section{Acknowledgments}

This work is supported by the BBSRC, EPSRC, MRC and Wellcome Trust. The C.B. laboratory is part of the Wellcome Trust Centre for CellMatrix research, University of Manchester, which is supported by core funding from the Wellcome Trust (grant number 088785/Z/09/Z). The authors wish to acknowledge the funding provided by the Biotechnology and Biological Sciences Research Council (BBSRC) to C.M., K.J. (BB/ M020630/1) and P.A. (BB/P000681/1) and by the Engineering and Physical Sciences Research Council (EPSRC) and Medical Research Council (MRC) Centre for Doctoral Training in Regenerative Medicine to A.K. (EP/L014904/1). The authors thank Alvéole for their correspondence and their after-sales support team. The authors thank Peter March and Roger Meadows from the Bioimaging Facility, University of Manchester for their help with the microscopy. The Bioimaging Facility microscopes used in this study were purchased with grants from BBSRC, Wellcome Trust and the University of Manchester Strategic Fund.

\section{References}

1. Alamdari, O. G., Seyedjafari, E., Soleimani, M., \& Ghaemi, N. Micropatterning of ECM Proteins on Glass Substrates to Regulate Cell Attachment and Proliferation. Avicenna Journal of Medical Biotechnology. 5 (4), 234-240 (2013)

2. Sunami, H., Yokota, I., \& Igarashi, Y. Influence of the pattern size of micropatterned scaffolds on cell morphology, proliferation, migration and F-actin expression. Biomaterials Science. 2 (3), 399-409, (2014).

3. Thery, M. Micropatterning as a tool to decipher cell morphogenesis and functions. Journal of Cell Science. 123 (Pt 24), 4201-4213, (2010).

4. Marino, A. et al. Two-photon polymerization of sub-micrometric patterned surfaces: investigation of cell-substrate interactions and improved differentiation of neuron-like cells. ACS Applied Materials \& Interfaces. 5 (24), 13012-13021 (2013).

5. Joo, S. et al. Effects of ECM protein micropatterns on the migration and differentiation of adult neural stem cells. Scientific Reports. 5 (2015).

6. Morgani, S. M., Metzger, J. J., Nichols, J., Siggia, E. D., \& Hadjantonakis, A. K. Micropattern differentiation of mouse pluripotent stem cells recapitulates embryo regionalized cell fate patterning. Elife. 7 (2018).

7. Javaherian, S., O'Donnell, K. A., \& McGuigan, A. P. A Fast and Accessible Methodology for Micro-Patterning Cells on Standard Culture Substrates Using Parafilm (TM) Inserts. Plos One. 6 (6) e2090910. (2011).

8. Smirnov, M. S., Cabral, K. A., Geller, H. M., \& Urbach, J. S. The effects of confinement on neuronal growth cone morphology and velocity. Biomaterials. 35 (25), 6750-6757 (2014).

9. Albert, P. J., \& Schwarz, U. S. Dynamics of Cell Ensembles on Adhesive Micropatterns: Bridging the Gap between Single Cell Spreading and Collective Cell Migration. PLOS Computational Biology. 12 (4), e1004863 (2016).

10. Evans, A. R. et al. Laminin and fibronectin modulate inner ear spiral ganglion neurite outgrowth in an in vitro alternate choice assay. Developmental Neurobiology. 67 (13), 1721-1730 (2007).

11. Nichol, R. H., Hagen, K. M., Lumbard, D. C., Dent, E. W., \& Gomez, T. M. Guidance of Axons by Local Coupling of Retrograde Flow to Point Contact Adhesions. Journal of Neuroscience. 36 (7), 2267-2282 (2016).

12. Burdick, J. A., Khademhosseini, A., \& Langer, R. Fabrication of gradient hydrogels using a microfluidics/photopolymerization process Langmuir. 20 (13), 5153-5156 (2004).

13. Schwartz, P. V. Molecular transport from an atomic force microscope tip: A comparative study of dip-pen nanolithography. Langmuir. 18 (10) 4041-4046 (2002).

14. Barbulovic-Nad, I. et al. Bio-microarray fabrication techniques--a review. Critical Reviews in Biotechnology. 26 (4), $237-259$ (2006).

15. Shafagh, R. Z., Vastesson, A., Guo, W. J., van der Wijngaart, W., \& Haraldsson, T. E-Beam Nanostructuring and Direct Click Biofunctionalization of Thiol-Ene Resist. Acs Nano. 12 (10), 9940-9946 (2018).

16. Kobayashi, J., Yamato, M., Itoga, K., Kikuchi, A., \& Okano, T. Preparation of microfluidic devices using micropatterning of a photosensitive material by a maskless, liquid-crystal-display projection method. Advanced Materials. 16 (22), 1997-+ (2004).

17. Bernard, A. et al. Printing patterns of proteins. Langmuir. 14 (9), 2225-2229 (1998).

18. Ruiz, S. A., \& Chen, C. S. Microcontact printing: A tool to pattern. Soft Matter. 3 (2), 168-177 (2007).

19. Qin, D., Xia, Y., \& Whitesides, G. M. Soft lithography for micro- and nanoscale patterning. Nature Protocols. 5 (3), $491-502$ (2010).

20. Fink, J. et al. Comparative study and improvement of current cell micro-patterning techniques. Lab Chip. 7 (6), $672-680$ (2007).

21. Strale, P. O. et al. Multiprotein Printing by Light-Induced Molecular Adsorption. Advanced Materials. 28 (10), $2024-2029$ (2016).

22. Belisle, J. M., Correia, J. P., Wiseman, P. W., Kennedy, T. E., \& Costantino, S. Patterning protein concentration using laser-assisted adsorption by photobleaching, LAPAP. Lab Chip. 8 (12), 2164-2167 (2008).

23. Belisle, J. M., Kunik, D., \& Costantino, S. Rapid multicomponent optical protein patterning. Lab Chip. 9 (24), $3580-3585$ (2009).

24. Heinz, W. F., Hoh, M., \& Hoh, J. H. Laser inactivation protein patterning of cell culture microenvironments. Lab Chip. 11 (19), $3336-3346$ (2011).

25. Azioune, A., Carpi, N., Tseng, Q., Thery, M., \& Piel, M. Protein Micropatterns: A Direct Printing Protocol Using Deep UVs. Microtubules: In Vivo. 97, 133-146 (2010).

26. Vignaud, T., Ennomani, H., \& Thery, M. Polyacrylamide hydrogel micropatterning. Methods in Cell Biology. 120, 93-116 (2014).

27. Waldbaur, A., Waterkotte, B., Schmitz, K., \& Rapp, B. E. Maskless projection lithography for the fast and flexible generation of grayscale protein patterns. Small. 8 (10), 1570-1578 (2012). 
28. Kang, J., Choi, J. C., Kim, M., Jung, H. R., \& Doh, J. Photopatterning with a printed transparency mask and a protein-friendly photoresist. Methods in Cell Biology. 119, 55-72 (2014).

29. Falconnet, D., Csucs, G., Grandin, H. M., \& Textor, M. Surface engineering approaches to micropattern surfaces for cell-based assays Biomaterials. 27 (16), 3044-3063 (2006).

30. Morlat, S., \& Gardette, J. L. Phototransformation of water-soluble polymers. Part II: photooxidation of poly(ethylene oxide) in aqueous solution. Polymer. 44 (26), 7891-7897 (2003).

31. Qi, Y., Wang, J. K., McMillian, M., \& Chikaraishi, D. M. Characterization of a CNS cell line, CAD, in which morphological differentiation is initiated by serum deprivation. Journal of Neuroscience. 17 (4), 1217-1225 (1997).

32. Shrirao, A. B. et al. A Versatile Method of Patterning Proteins and Cells. Journal of Visualized Experiments. (120) (2017).

33. Pankov, R., \& Momchilova, A. Fluorescent labeling techniques for investigation of fibronectin fibrillogenesis (labeling fibronectin fibrillogenesis). Methods in Molecular Biology. 522, 261-274 (2009).

34. Dertinger, S. K., Jiang, X., Li, Z., Murthy, V. N., \& Whitesides, G. M. Gradients of substrate-bound laminin orient axonal specification of neurons. Proceedings of the National Academy of Sciences of the United States of America. 99 (20), 12542-12547 (2002).

35. Chelli, B. et al. Neural cell alignment by patterning gradients of the extracellular matrix protein laminin. Interface Focus. 4 (1), 20130041 (2014).

36. Tang, Y., Qiu, Q. F., Zhang, F. L., Xie, M., \& Huang, W. H. Quantifying orientational regeneration of injured neurons by natural product concentration gradients in a 3D microfluidic device. Lab Chip. 18 (6), 971-978 (2018).

37. Srinivasan, P., Zervantonakis, I. K., \& Kothapalli, C. R. Synergistic effects of 3D ECM and chemogradients on neurite outgrowth and guidance: a simple modeling and microfluidic framework. PLoS One. 9 (6), e99640 (2014).

38. de Luca, A. C., Faroni, A., \& Reid, A. J. Dorsal root ganglia neurons and differentiated adipose-derived stem cells: an in vitro co-culture model to study peripheral nerve regeneration. Journal of Visualized Experiments. (96) (2015).

39. Khadpekar, A. J., Khan, M., Sose, A., \& Majumder, A. Low Cost and Lithography-free Stamp fabrication for Microcontact Printing. Scientific Reports. 9 (1), 1024 (2019)

40. Delepine, C. et al. Altered microtubule dynamics and vesicular transport in mouse and human MeCP2-deficient astrocytes. Human Molecular Genetics. 25 (1), 146-157 (2016).

41. Decock, J., Schlenk, M., \& Salmon, J. B. In situ photo-patterning of pressure-resistant hydrogel membranes with controlled permeabilities in PEGDA microfluidic channels. Lab Chip. 18 (7), 1075-1083 (2018).

42. Stoecklin, C. et al. A New Approach to Design Artificial 3D Microniches with Combined Chemical, Topographical, and Rheological Cues. Advanced Biosystems. 2 (7) (2018).

43. Toraille, L. et al. Optical Magnetometry of Single Biocompatible Micromagnets for Quantitative Magnetogenetic and Magnetomechanical Assays. Nano Letters. (2018).

44. Theodoly, O. et al. Live nanoscopic to mesoscopic topography reconstruction with an optical microscope for chemical and biological samples. PLoS One. 13 (12), e0207881 (2018).

45. Ermis, M., Antmen, E., \& Hasirci, V. Micro and Nanofabrication methods to control cell-substrate interactions and cell behavior: A review from the tissue engineering perspective. Bioactive Materials. 3 (3), 355-369 (2018).

46. von Philipsborn, A. C. et al. Microcontact printing of axon guidance molecules for generation of graded patterns. Nature Protocols. 1 (3), 1322-1328 (2006).

47. Ricoult, S. G., Kennedy, T. E., \& Juncker, D. Substrate-bound protein gradients to study haptotaxis. Frontiers in Bioengineering and Biotechnology. 3, 40 (2015).

48. Bietsch, A., \& Michel, B. Conformal contact and pattern stability of stamps used for soft lithography. Journal of Applied Physics. 88 (7), 4310-4318 (2000).

49. Hui, C. Y., Jagota, A., Lin, Y. Y., \& Kramer, E. J. Constraints on microcontact printing imposed by stamp deformation. Langmuir. 18 (4), 1394-1407 (2002).

50. Sharp, K. G., Blackman, G. S., Glassmaker, N. J., Jagota, A., \& Hui, C. Y. Effect of stamp deformation on the quality of microcontact printing: theory and experiment. Langmuir. 20 (15), 6430-6438 (2004).

51. Delamarche, E., Schmid, H., Michel, B., \& Biebuyck, H. Stability of molded polydimethylsiloxane microstructures. Advanced Materials. 9 (9), 741-746 (1997).

52. Perl, A., Reinhoudt, D. N., \& Huskens, J. Microcontact Printing: Limitations and Achievements. Advanced Materials. 21 (22), $2257-2268$ (2009).

53. Chakra, E. B., Hannes, B., Dilosquer, G., Mansfield, C. D., \& Cabrera, M. A new instrument for automated microcontact printing with stamp load adjustment. Review of Scientific Instruments. 79 (6) (2008).

54. Pasturel, A., Strale, P., Studer, V. Tailoring 3D cell culture templates with common hydrogels. bioRxiv. (2019). 